\title{
Hard QCD Measurements in ATLAS
}

\author{
Rainer Stamen*i \\ Kirchhoff-Institut für Physik \\ Universität Heidelberg \\ E-mail: stamen@kip.uni-heidelberg.de
}

Proton-Proton collisions at the LHC offer the possibility to study perturbative QCD at unprecedentedly small distances. Various observables involving jets enable detailed tests of current MC generators as well as NLO calculations in a new region of phase space opened by the large centreof-mass energy provided by the LHC. The extension of the kinematic reach w.r.t. the Tevatron for inclusive jet and multijet transverse momentum spectra and for dijet mass spectra was possible already in the initial phase of data taking with a relatively small amount of integrated luminosity of $15-300 \mathrm{nb}^{-1}$ due to the large QCD cross section in proton-proton interactions with a centre-ofmass energy of $\sqrt{s}=7 \mathrm{TeV}$. Here we review Quantum Chromodynamics (QCD) measurements involving jets from the initial data taking phase of LHC which are well described in the framework of pertubative QCD. In additon to proton-proton interactions, jets have also been studied in the first run of heavy ion collisions where a significant dijet asymmetry has been observed.

Workshop on Discovery Physics at the LHC-Kruger 2010

December 05-10, 2010

Kruger National Park, Mpumalanga, South Africa

\footnotetext{
* Speaker.

${ }^{\dagger}$ On behalf of the ATLAS collaboration
} 


\section{Introduction}

The LHC [1] started colliding protons at a centre-of-mass energy of $\sqrt{s}=7 \mathrm{TeV}$ early in 2010. Here we review Quantum Chromodynamics (QCD) measurements made with the ATLAS detector [2] during the initial data-taking period. Although the data sample is modest, the large cross section for QCD processes already allows significant tests of QCD phenomenology. Such results are important in themselves and for searches for new physics phenomena and their potential interpretation.

\subsection{QCD studies at the LHC}

The cross section for proton-proton (pp) interactions yielding a final state $X$ can be calculated as

$$
\sigma_{p p \rightarrow X}=\sum_{a, b} \int_{0}^{1} d x_{1} d x_{2} f_{a}\left(x_{1}, \mu_{F}^{2}\right) f_{b}\left(x_{2}, \mu_{F}^{2}\right) \times \hat{\sigma}_{a b \leftarrow X}\left(x_{1}, x_{2}, \alpha_{S}\left(\mu_{R}^{2}\right), \mu_{R}^{2}, \mu_{F}^{2}\right)
$$

where the matrix element $\hat{\sigma}_{a b \leftarrow X}\left(x_{1}, x_{2}, \alpha_{S}\left(\mu_{R}^{2}\right), \mu_{R}^{2}, \mu_{F}^{2}\right)$ depicts the partonic hard scattering process. It depends on the Bjorken scaling variables $x_{1 / 2}$, i.e. the momentum fractions of the initial state partons of the colliding protons, the strong coupling constant $\alpha_{s}$ and the renormalisation and factorisation scales $\mu_{R}$ and $\mu_{F}$ respectively. The matrix element can be calculated in the framework of perturbative QCD (pQCD) and is dominated by $2 \rightarrow 2$ partonic scattering processes. It is convoluted with the parton density functions $f_{a / b}\left(x_{1 / 2}, \mu_{F}^{2}\right)$ which describe the partonic content of the proton. Such calculations are typically performed at next-to-leading order (NLO) in pQCD and yield cross sections for partonic final states. The calculations implemented in $\mathrm{MC}$ generators are typically based on leading order (LO) QCD calculations.

QCD studies thus give access to various aspects of a pp scattering process. These are the parton density functions, the hard matrix element, initial and final state QCD radiation, parton showers of the final state partons, hadronisation and the underlying event. The measurements can be used for detailed tests of the QCD calculations and the implementation of various models for the non-perturbative components of the theoretical predictions. Furthermore a precise knowledge of most of these components is important for many of the searches for new physics since QCD processes often constitute one of the dominant backgrounds for such measurements.

\subsection{The ATLAS Experiment and the Data Sets}

The ATLAS experiment at the LHC is a multipurpose detector designed to cover a broad range of physics measurements, in particular the search for the Higgs boson and phenomena beyond the Standard Model. The detector system most relevant for the results presented here is the fine-grained sampling calorimeter system which uses liquid argon as the active medium for the electromagnetic calorimeters and scintillator tiles or liquid argon in the hadronic calorimeters.

The LHC started colliding protons at $\sqrt{s}=7 \mathrm{TeV}$ at the end of March 2010 with only a few bunches of protons in the machine and a low number of protons per bunch as well as weak focusing of the beams in the interaction region. During the course of the year all these parameters have 
been subsequently optimised and the instantaneous luminosity raised by more than five orders of magnitude from about $10^{27} \mathrm{~cm}^{-2} \mathrm{~s}^{-1}$ to $2 \times 10^{32} \mathrm{~cm}^{-2} \mathrm{~s}^{-1}$.

The trigger was continuously adjusted to the increasing collision rates and to keep the output rate at about $300 \mathrm{~Hz}$. The primary trigger required hits in scintillators which are located on each side of the experiment just in front of the calorimeter and formed the sole trigger requirement during the initial phase of data taking. With increasing luminosity the standard ATLAS jet triggers were added. These used a hardware-based calorimeter jet trigger and a higher level trigger decision formed in a PC farm which ran reconstruction and identification algorithms similar to those used in offline analyses. The results presented here are based on small data sets ranging from integrated luminosities of $17 \mathrm{nb}^{-1}$ to about $300 \mathrm{nb}^{-1}$.

\subsection{Jet finding and Jet Energy Scale uncertainty}

Most of the results presented here are based on jets which are reconstructed using a standard anti-kt algorithm [3] with a resolution parameter $\mathrm{R}=0.6$. The inputs to the anti-kt jet algorithm are clusters of calorimeter cells seeded by cells with energy that is significantly above the measured noise [4]. The main systematic uncertainty in the measurements presented here constitutes the jet energy scale uncertainty which is about 7\% [4]. A custom jet-finding algorithm based on calorimeter towers was used in the analysis of the heavy ion data

\section{Jet measurements in proton-proton interactions}

In this section we present various measurements involving jets. After the investigation of jet shapes which tests the detailed modelling of jets in our MC generators, we present the measurement of the inclusive jet cross section. We then present more exclusive measurements such as the cross sections for dijet and multijet observables. The study of angular decorrelations probes higher order partonic radiation with a rather simple observable only involving the two most energetic jets in an event. All these measurements are compared with NLO calculations corrected for soft effects and MC generators involving different versions of soft QCD models for hadronisation and the underlying event.

Various selection criteria are common to all the presented analyses. Events are selected which pass the first level trigger jet selection. This trigger condition is $100 \%$ efficient for events where the leading jet has a transverse momentum of at least $60 \mathrm{GeV}$ which is therefore applied as a minimal requirement for the leading jet. A special cleaning is performed to reject jets originating from calorimeter noise or jets induced by energy depositions of cosmic muons. In order to select only jets from pp collisions, a reconstructed vertex built from at least five reconstructed tracks is required. These conditions reduce all types of background to a negligible level such that all reconstructed jets can be interpreted as originating from a hard QCD scattering process in the pp interaction.

\subsection{Jet Shapes}

Jets are formed in the phase after the hard scattering took place and their shape depends mainly on the parton shower, i.e. hard partonic radiation from the produced final state parton and hadronisation processes. The parton shower can be calculated in the framework of pQCD whereas the hadronisation step is modelled phenomenologically. A measurement of the jet shape therefore 
serves as a good test of state of the art MC generators which implement the theoretical predictions and which have been tuned using data taken at other high energy colliders.

The variable measured here is the differential jet shape, defined as the fraction $\rho(r)=p_{T}^{r} / p_{T}^{R}$, where $p_{T}^{R}$ is the transverse momentum of calorimeter clusters within the radius $R$ of the jet centre, and $p_{T}^{r}$ is the transverse momentum contained within a ring of thickness $\Delta r=0.1$ at a radius $r$ around the jet centre. The measurement shown in Figure 1 is compared with MC simulation based on the PYTHIA [5] and HERWIG [6] MC programs. Both programs describe the data reasonably well with PYTHIA producing slightly narrower jets than HERWIG.

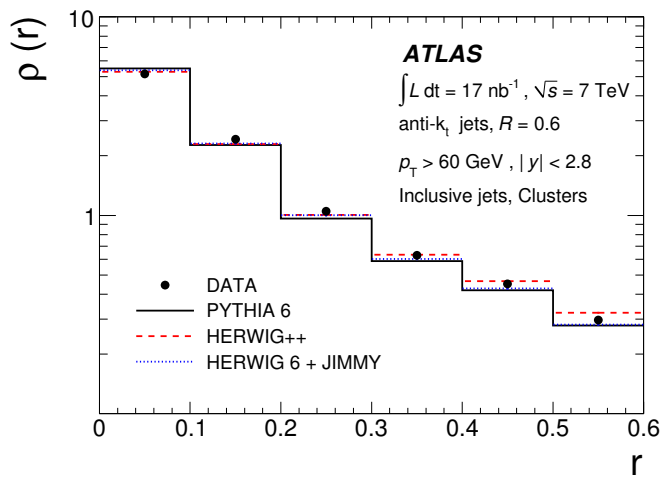

Figure 1: Differential Jet Shape $\rho(r)$ [4].

\subsection{Inclusive Jet Cross Section}

The measurement of the inclusive jet cross Section constitutes a very important test of the theory of strong interactions. The single differential cross section as a function of the jet transverse momentum varies over many orders of magnitude in the available phase space. Here we report the first ATLAS measurement using a small data set which corresponds to an integrated luminosity of $17 \mathrm{nb}^{-1}$. In the phase space available for this measurement effects from new physics are not expected, which makes it a very important benchmark measurement to test theoretical calculations as well as tools like MC generators and the measurement procedures and detector modelling.

Jets were selected as described above with a transverse momentum of at least $p_{T}>60 \mathrm{GeV}$ within the rapidity range $|y|<2.8$. The measured jet $p_{T}$ is corrected to the particle level scale [4] using an average correction, computed as a function of jet transverse momentum and pseudorapidity, and extracted from MC simulation. These correction factors are typically below $20 \%$. Various systematic uncertainties have been studied and the dominant systematic uncertainty, on jet energy scale, is less than $7 \%$ in the kinematic range of these measurements.

Figure 2 shows the single differential inclusive jet cross section as function of jet transverse momentum [4]. The data are compared to a NLO pQCD calculation which has been corrected for

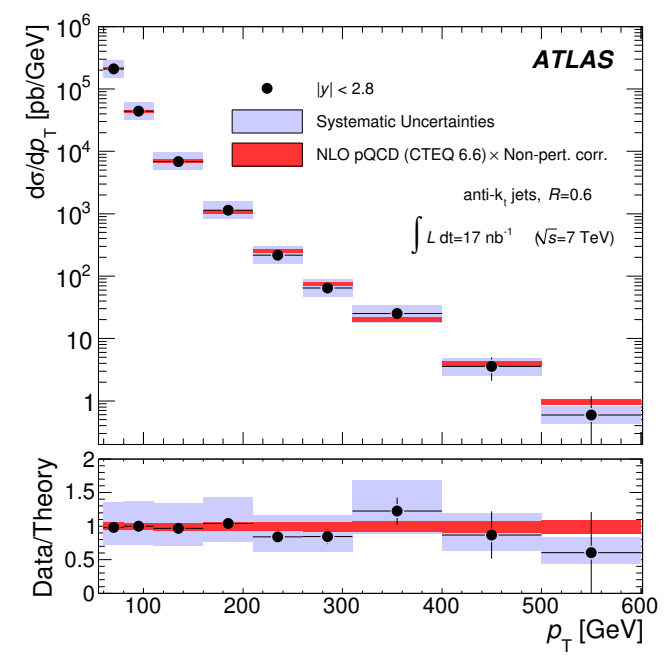

Figure 2: Inclusive Jet Cross as function of jet transverse momentum [4]. soft effects during the hadronisation phase using a MC generator. A very good agreement between the data points and the theoretical predictions can be observed. In addition, the double differential inclusive jet cross section was measured as a function of transverse momentum and rapidity with the same conclusion. 


\subsection{Dijet Cross Section}

The partonic subprocess in hard pp interactions is dominated by $2 \rightarrow 2$ scattering processes yielding a majority of events with two jets in the final state. The measurement of the cross section as a function of the invariant mass of the dijet system is of particular interest since this observable is sensitive to processes such as contact interactions or even resonance production of exotic particles. Both will show up as prominent discrepancies when data are compared to theory predictions based solely on QCD.

The analysis is very similar to the inclusive jet cross section measurement except that subleading jets are accepted above a lower transverse momentum threshold of $p_{T}>30 \mathrm{GeV}$. In Figure 3 the cross section for dijet production is

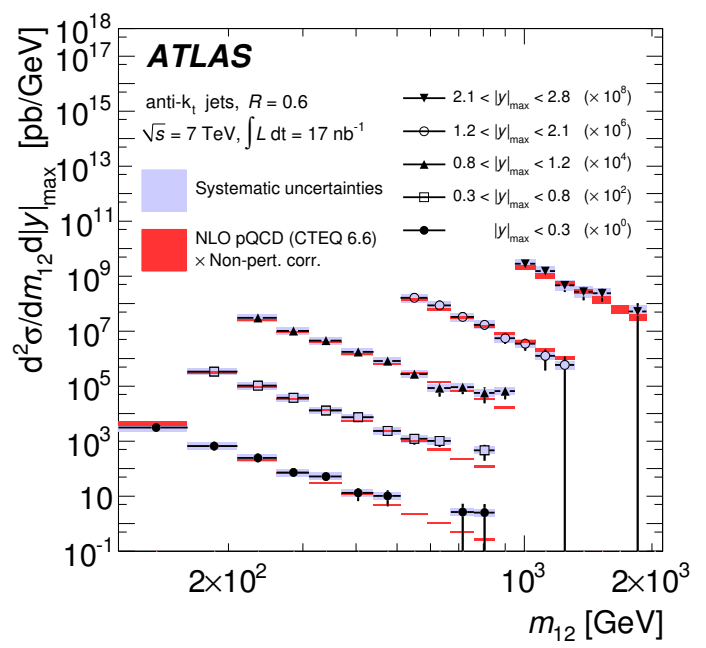

Figure 3: Cross Section for dijet production as a function of the invariant mass [4]. shown as a function of the invariant mass of the dijet system for various regions of the rapidity of the leading jet. The data are compared to NLO pQCD calculations corrected for soft effects. Very good agreement is seen in this comparison, and also when comparing data with MC simulations. Furthermore it can be observed that, even in this small sample of data, the data reach invariant masses of up to $2 \mathrm{TeV}$ which is above the reach of the Tevatron.

\subsection{Multijet Measurements}

The measurement of the multijet cross section constitutes an even stronger test of QCD. In pp collisions, events with more than 2 jets can be produced either directly in the hard scattering processes or during the parton shower by the emission of a parton from the outgoing partons of the hard scattering. Figure 4 shows the transverse momentum spectrum for the fourth subleading jet in a multijet environment [7]. The data are compared to two very different MC generators. PYTHIA simulates dijets events in which any additional subleading jets are mainly produced by gluon radiation from the final state partons, whereas Alpgen [8] also simulates final states with more than two partons in the final state of the hard scattering processes. The PYTHIA pre-

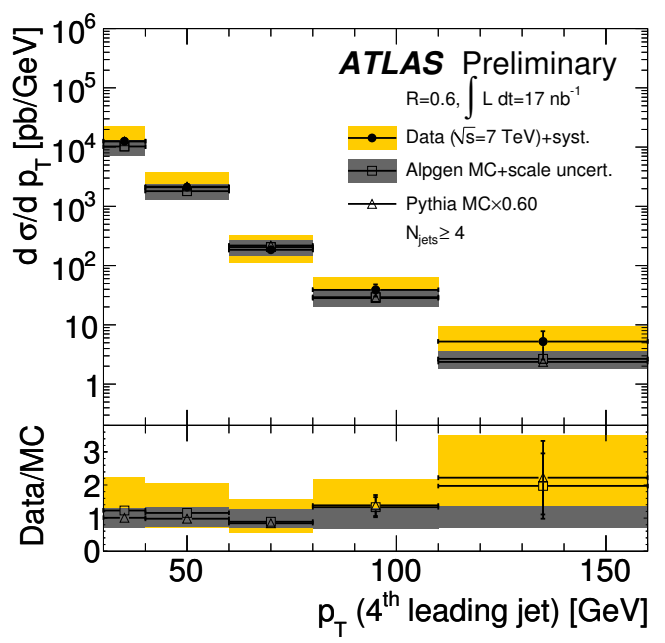

Figure 4: Transverse momentum spectrum for the fourth leading jet [7]. dictions have been scaled by a global factor to describe the data, which is not unexpected since its predictions are based on a LO calculation. It 
however describes the shape of the data quite well. Alpgen describes the shape of the data as well as the magnitude of the cross section.

\subsection{Dijet Angular Decorrelation}

The measurements of dijet angular decorrelations constitutes a very interesting method to access multi parton production (either in the hard scattering process or via gluon radiation in the subsequent parton shower) using only the two hardest jets per event. The majority of events consist of dijet signatures where both jets are back to back in the transverse plane due to energy momentum conservation. This can be quantified by the observable $\Delta \phi$ which is the difference of the scattering angles of the two leading in $p_{T}$ jets in the transverse plane perpendicular

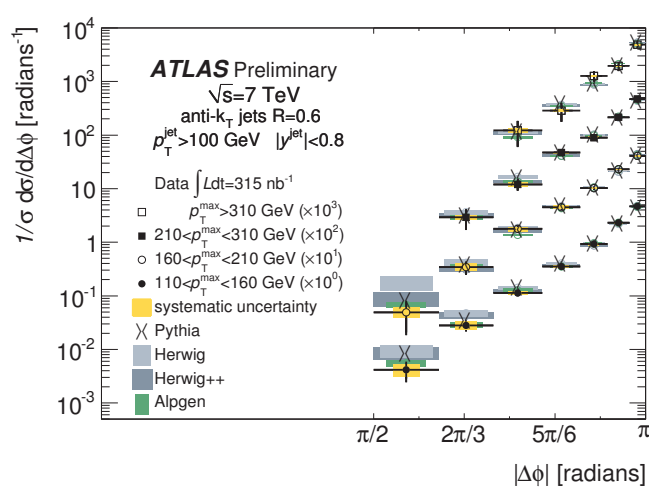

Figure 5: Dijet Angular Decorrelation [9]. to the proton beam axis. A value of $\Delta \phi=\pi$ is expected for perfect dijet events. Deviations from this value are expected from detector resolution effects and more importantly for events with additional radiation or jet production where the system of the two leading jets is balanced against the additional activity in the event. Figure 5 shows the measurement of the the azimuthal decorrelation for various regions of rapidity of the leading jet [9]. The transverse momenta of the two leading jets are required to be above $p_{T 1}>110 \mathrm{GeV}$ and $p_{T 1}>100 \mathrm{GeV}$ respectively. The data are compared to various MC generators. A reasonable description is given by all MC generators within the current measurement uncertainties. Although PYTHIA and Alpgen seem to describe the data better than HERWIG, a more precise measurement is needed to draw final conclusions.

\section{Dijet Asymmetry in Lead-Lead Collisions}

Shortly before this conference, the LHC collided heavy ions for the first time at a per-nucleon center of mass energy of $2.76 \mathrm{TeV}$. A dataset with an integrated luminosity of $1.7 \mu \mathrm{b}^{-1}$ has been collected. The study of jets in heavy ion collisions serves as a tool to study the propagation of highly energetic partons in a dense partonic media. Jets are dominantly produced in pairs. When one of the two jets propagates a long distance through a hot medium it can be modified by interactions with the medium through scattering and radiation processes and it can thus lose a significant amount of energy. In order to quantify this effect the asymmetry $A_{J}$ has been measured. This is defined as $A_{J}=\left(E_{T 1}-E_{T 2}\right) /\left(E_{T 1}-E_{T 2}\right)$ where $E_{T 1}$ and $E_{T 2}$ denote the transverse energies of the two leading jets in the selected heavy ion events. Jets have been reconstructed using an anti-kt algorithm with a radius parameter $R=0.4$. The inputs to this algorithm are "towers" of calorimeter cells of size $\Delta \eta \times \Delta \phi=0.1 \times 0.1$. The asymmetry has been measured for various collision centralities, where centrality is a measure of the number of interacting nucleons in the heavy ion collision. As shown in Figure 6 a significant asymmetry can be observed for large centrality events which can be 
interpreted as jet modification for events where the initial partons propagate a long distance through a dense partonic medium [10].
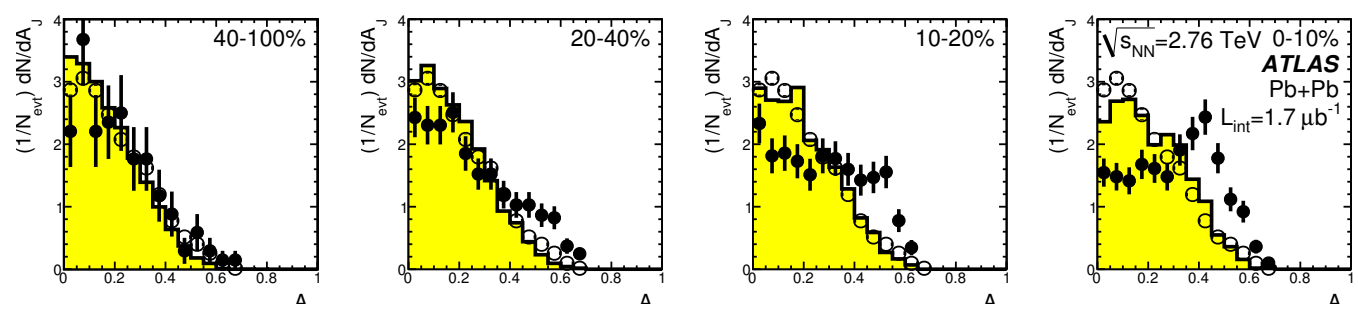

Figure 6: Measurement of the Dijet Asymmetry $A_{J}$ for different centralities [10].

\section{Conclusions}

We have presented several measurements using the data from the initial data taking phase of the ATLAS experiment and studied properties of processes which involve the strong force. Properties of hadronic jets have been determined and their cross sections have been measured. Good agreement has been found with NLO QCD calculations and state of the art MC generators, including phase space regions not covered by earlier experiments. This is a decisive test of QCD itself and a very important prerequisite for searches for new physics. The study of dijet systems in heavy ion collisions lead to the discovery of significant jet modifications in dense partonic media.

\section{References}

[1] L. Evans and P. Bryant (editors), JINST 3 (2008) S08001.

[2] G. Aad et al. [ATLAS Collaboration], JINST 3 (2008) S08003.

[3] M. Cacciari, G. P. Salam and G. Soyez, JHEP 0804, 063 (2008).

M. Cacciari and G. P. Salam, Phys. Lett. B 641, 57 (2006).

[4] G. Aad et al. [Atlas Collaboration], Eur. Phys. J. C 71, 1512 (2011).

[5] T. Sjostrand, S. Mrenna and P. Z. Skands, JHEP 0605 (2006) 026.

[6] G. Corcella et al., JHEP 0101 (2001) 010.

[7] The ATLAS Collaboration, "Measurements of multijet production cross sections in proton-proton collisions at $7 \mathrm{TeV}$ center-of-mass energy with the ATLAS Detector," ATLAS-CONF-2010-084.

[8] M. L. Mangano, M. Moretti, F. Piccinini, R. Pittau and A. D. Polosa, JHEP 0307 (2003) 001.

[9] The ATLAS Collaboration, "Azimuthal Decorrelations in Dijet Events at $\sqrt{s}=7 \mathrm{TeV}$," ATLAS-CONF-2010-083.

[10] G. Aad et al. [Atlas Collaboration], Phys. Rev. Lett. 105, 252303 (2010). 